POS PROCEEDINGS

\title{
Quark and gluon jet functions at three loops in QCD
}

\section{Pulak Banerjee}

Paul Scherrer Institut, Forschungsstrasse 111, CH-5232 Villigen PSI, Switzerland

E-mail: pulak.banerjee@psi.ch

\section{Prasanna K. Dhani*}

INFN, Sezione di Firenze, I-50019 Sesto Fiorentino, Florence, Italy

E-mail: dhani@fi.infn.it

\section{Ravindran}

The Institute of Mathematical Sciences, Taramani, Chennai 600113, India

Homi Bhabha National Institute, Training School Complex, Anushakti Nagar, Mumbai 400085,

India

E-mail: ravindra@imsc.res.in

\begin{abstract}
We present the first result on the three-loop parton (quark/gluon) jet function in perturbative quantum chromodynamics using already known three-loop coefficient functions for deep-inelastic scattering via the exchange of a virtual photon that couples to quarks or a scalar that couples to gluons. While the gluon jet function is a brand new result emerging from this article, the result for the quark jet function provides an independent check to a more recent calculation. These jet functions being universal ingredients in the Soft-collinear effective theory framework, will play an important role in the phenomenological studies at the Large Hadron Collider, such as resummation of jet observables and also in $\mathrm{N}$-jettiness subtraction method.
\end{abstract}

14th International Symposium on Radiative Corrections (RADCOR2019)

9-13 September 2019

Palais des Papes, Avignon, France

\footnotetext{
*Speaker.
} 


\section{Introduction}

In this precision era of the standard model (SM) of particle physics, it is important to accurately measure various observables and compare them with the precise theoretical predictions that uses state-of-the-art modern techniques. In addition such studies are of paramount importance to understand the SM background to constrain any physics beyond the SM. In particular, processes such as production of lepton pairs, vector bosons and multi-jets at the Large Hadron Collider (LHC) play an important role in these studies. In addition, understanding of the jets will shed light on the underlying structure of quantum chromodynamics (QCD).

The hadronic cross sections in the processes such as the lepton-nucleon deep-inelastic scattering (DIS) or in the Drell-Yan processes factorize into hard and soft plus virtual (SV) parts. The hard part is associated with the physics of large momentum transfer $Q$; the soft part of the SV cross section describes the interactions associated with emission of soft and collinear partons. Soft-collinear effective theory (SCET) [1, 2, 3, 4, 5, 6] captures the physics of soft and collinear dynamics of these processes at high energies through the soft and jet functions. The jet functions explain the propagation of collinear partons inside jets and the soft functions mediate low-energy interaction between jets. In SCET, the quark and gluon soft as well as jet functions have been computed to higher orders in perturbation theory. The quark and the gluon soft functions are already known to the three-loop level; see [7] and references therein. For the quark jet function, results were known up to the two-loop level for some time (see $[8,9,10]$ ), and the results at three loops were computed recently in [11]. On the other hand, only one- [12] and two-loop [13] results are known for the gluon jet function.

Our goal in this article is to demonstrate an intriguing connection between jet functions in SCET and coefficient functions of DIS cross sections computed in perturbative QCD. We exploit this novel connection to obtain quark and gluon jet functions up to three-loop level using the known state-of-the-art three-loop coefficient functions in the context of DIS [14, 15]. To achieve this, we exploit the Sudakov (KG) equation that the virtual part (form factor) of the cross section satisfy, renormalization group invariance, factorization theorem and use various three-loop results. We use the framework developed in $[16,17,18,19,20]$ which describes resummation of soft gluons to all orders in QCD perturbation theory. In [17], it was shown that DIS cross section in the threshold limit factorizes into the square of ultraviolet (UV) renormalized form factor, soft plus jet (SJ) function and the mass factorization kernels. The SJ function has a universal structure and it depends only on the nature of external states namely quark or gluon states. In addition, it satisfies KG type differential equation similar to the form factors. Factorization properties of the cross section and renormalization group invariance can be used to unravel the structure of these SJ functions to all orders in perturbation theory. Upon identifying the finite part of the square of the form factor with the hard matching coefficient in the SCET framework, the finite part of SJ function can be shown to coincide with the corresponding jet function. The latter identification is simply due to the process-independent nature of both the SJ function and the jet function. We first demonstrate that the quark jet function known up to the three-loop level agrees with the finite part of quark SJ function obtained from the coefficient function [14] and proceed in the similar way to 
obtain the corresponding gluon jet function from [15].

\section{Theoretical framework and details of the calculation}

The inclusive cross section for the scattering of a lepton with a hadron in the DIS is given by

$$
\sigma^{I}\left(x, Q^{2}\right)=\sigma_{B}^{I}\left(\mu_{R}^{2}\right) \sum_{a=q, \bar{q}, g} \int_{x}^{1} \frac{d z}{z} f_{a}\left(\frac{x}{z}, \mu_{F}^{2}\right) \Delta_{a}^{I}\left(a_{s}, z, Q^{2}, \mu_{R}^{2}, \mu_{F}^{2}\right) .
$$

The scaling variables at the hadronic and partonic level are given by $x=\frac{-q^{2}}{2 P \cdot q}, z=\frac{-q^{2}}{2 p \cdot q}$ respectively. $P, p$ and $q$ are the momenta of the hadron, fraction of the parent hadron momentum and the intermediate off-shell particle respectively. For DIS we have $Q^{2}=-q^{2}$. The parton distribution function is given by $f_{a}\left(x, \mu_{F}^{2}\right)$ with the momentum fraction $x$ of the hadron at the factorization scale $\mu_{F}$. The particle probing the structure of the hadron can be a photon $(I=q)$ or the scalar particle $(I=g)$. The perturbatively computed UV and infrared (IR) finite part of the partonic cross section, called the coefficient function, is given by $\Delta_{a}^{I}\left(a_{s}, z, Q^{2}, \mu_{R}^{2}, \mu_{F}^{2}\right)$, where $\mu_{R}$ denotes the UV renormalization scale. The Born contribution $\sigma_{B}^{I}$ is chosen in such a way that $\Delta_{a}^{I}$ to lowest order in perturbation theory is equal to $\delta(1-z)$. The UV renormalized strong coupling constant is $a_{s}=g_{s}^{2}\left(\mu_{R}^{2}\right) / 16 \pi^{2}$ is written in terms of bare coupling constant $\hat{a}_{s}=\hat{g}_{s}^{2} / 16 \pi^{2}$ as $\hat{a}_{s}\left(\mu^{2}\right) S_{\varepsilon}=a_{s}\left(\mu_{R}^{2}\right)\left(\mu^{2} / \mu_{R}^{2}\right)^{\varepsilon / 2} Z\left(a_{s}\left(\mu_{R}^{2}\right)\right)$. The scale $\mu$ keeps $\hat{g}_{s}$ dimensionless in $n=4+\varepsilon$ space-time dimensions. $S_{\varepsilon}$ is the spherical factor defined as $S_{\varepsilon}=\exp \left[\left(\gamma_{E}-\ln 4 \pi\right) \frac{\varepsilon}{2}\right]$, where $\gamma_{E}$ is the Euler-Mascheroni constant. $Z\left(a_{s}\left(\mu_{R}^{2}\right)\right)$ is the renormalization term for the coupling constant.

The infrared safe coefficient functions $\Delta_{a}^{I}$ get contributions from both soft gluons as well as from hard partons. We write them as sum of contributions from SV and the remaining hard part:

$$
\Delta_{a}^{I}\left(a_{s}, z, Q^{2}, \mu_{F}^{2}, \mu_{R}^{2}\right)=\Delta^{I, \mathrm{SV}}\left(a_{s}, z, Q^{2}, \mu_{F}^{2}, \mu_{R}^{2}\right)+\Delta_{a}^{I, \text { hard }}\left(a_{s}, z, Q^{2}, \mu_{F}^{2}, \mu_{R}^{2}\right) .
$$

The SV part of the cross section consists only of plus distributions $\mathscr{D}_{i}(z)=\left[\frac{\ln ^{i}(1-z)}{1-z}\right]_{+}$and $\delta(1-z)$. In the soft limit $z \rightarrow 1$ these distributions give dominant contributions to the hadronic cross section after they are convolved with the parton distribution functions as expressed through (2.1). As discussed in [16] the SV part of the coefficient function can be shown to factorize in terms of the square of the UV renormalized virtual contributions, SJ function appropriately convoluted with mass factorization kernels as

$$
\begin{aligned}
\Delta^{I, \mathrm{SV}}\left(z, Q^{2}\right) & =\left(Z^{I}\left(\hat{a}_{s}, \mu_{R}^{2}, \mu^{2}, \varepsilon\right)\right)^{2}\left|\hat{\mathscr{F}}^{I}\left(\hat{a}_{s}, Q^{2}, \mu^{2}, \varepsilon\right)\right|^{2} \delta(1-z) \otimes \mathscr{C} e^{2 \Phi_{\mathrm{SJ}}^{I}\left(\hat{a}_{s}, Q^{2}, \mu^{2}, z, \varepsilon\right)} \\
& \otimes \Gamma_{I I}^{-1}\left(\hat{a}_{s}, \mu^{2}, \mu_{F}^{2}, z, \varepsilon\right) .
\end{aligned}
$$

The symbol $\mathscr{C}$ denotes convolution operation and $\otimes$ indicates the Mellin convolution, which convolutes with respect to the variable $z$. As we are interested in evaluating the SV part of the cross sections, we neglect all the regular functions that come from different convolutions. In (2.3), the overall renormalization constant, $Z^{I}\left(\hat{a}_{s}, \mu_{R}^{2}, \mu^{2}, \varepsilon\right)$, for $I=g$ can be obtained from that of Higgsgluon effective operator and the exact form can be written in term of the anomalous dimension $\gamma_{g}$, known to the three-loop level [21]. For $I=q$, one finds $Z^{q}\left(\hat{a}_{s}, \mu_{R}^{2}, \mu^{2}, \varepsilon\right)=1$ to all orders due 
to vector current conservation of the respective interaction vertex. $\Gamma_{I I}\left(\hat{a}_{s}, \mu^{2}, \mu_{F}^{2}, z, \varepsilon\right)$ is the mass factorization kernel, known fully up to the three-loop level $[22,23]$ and in the large $n_{f}$ limit at the four-loop level [24]. The quantity $\hat{\mathscr{F}}^{I}\left(\hat{a}_{s}, Q^{2}, \mu^{2}, \varepsilon\right)$ is the bare form factor which satisfies the $\mathrm{KG}$ equation, where the latter is a consequence of factorization, gauge and renormalization group invariances [25, 26, 27, 28]. Its general solution up to four loops can be found in $[29,16]$ in terms of the universal three-loop cusp $\left(A^{I}\right)$ [22, 23, 30, 31, 32], collinear $\left(B^{I}\right)$ [22, 23], soft $\left(f^{I}\right)$ [33, 23] anomalous dimensions known to three-loop level and some form factor dependent constants. The quantity $\Phi_{\mathrm{SJ}}^{I}\left(\hat{a}_{s}, Q^{2}, \mu^{2}, z, \varepsilon\right)$ in (2.3) is the SJ function which contains singular as well as finite parts due to soft gluons and collinear parton emissions. In [16, 17], it was shown that by demanding finiteness of $\Delta^{I, \mathrm{SV}}, \Phi_{\mathrm{SJ}}^{I}\left(\hat{a}_{s}, Q^{2}, \mu^{2}, z, \varepsilon\right)$ can also be shown to satisfy a Sudakov-type differential equation and the solution is found to be

$$
\Phi_{\mathrm{SJ}}^{I}=\sum_{i=1}^{\infty} \hat{a}_{S}^{i} S_{\varepsilon}^{i}\left(\frac{Q^{2}(1-z)}{\mu^{2}}\right)^{i \frac{\varepsilon}{2}} \frac{i \varepsilon}{2(1-z)} \hat{\phi}_{\mathrm{SJ}}^{I,(i)}(\varepsilon),
$$

with

$$
\hat{\phi}_{\mathrm{SJ}}^{I,(i)}(\varepsilon)=\frac{1}{i \varepsilon}\left(\bar{K}^{I,(i)}(\varepsilon)+\bar{G}_{\mathrm{SJ}}^{I,(i)}(\varepsilon)\right) .
$$

Expressing $\bar{K}^{I}=\sum_{i=1}^{\infty} \hat{a}_{s}^{i}\left(\mu_{R}^{2} / \mu^{2}\right)^{i \frac{\varepsilon}{2}} S_{\varepsilon}^{i} \bar{K}^{I,(i)}$, the coefficients $\bar{K}^{I,(i)}(\varepsilon)$ can be written in terms of $A_{i}^{I}$, beta function of QCD, $\beta_{i}[34]$ and $\bar{G}_{\mathrm{SJ}}^{I,(i)}(\varepsilon)$ given by

$$
\sum_{i=1}^{\infty} \hat{a}_{s}^{i}\left(\frac{Q_{z}^{2}}{\mu^{2}}\right)^{i \frac{\varepsilon}{2}} S_{\varepsilon}^{i} \bar{G}_{\mathrm{SJ}}^{I,(i)}(\varepsilon)=\sum_{i=1}^{\infty} a_{s}^{i}\left(Q_{z}^{2}\right) \overline{\mathscr{G}}_{i, \mathrm{SJ}}^{I}(\varepsilon),
$$

with $Q_{z}^{2}=Q^{2}(1-z)$, can be expressed in terms of the $B^{I}-, f^{I}-$ and $\varepsilon$-dependent part in the following way: $\overline{\mathscr{G}}_{i, \mathrm{SJ}}^{I}=-\left(B_{i}^{I}+f_{i}^{I}\right)+C_{i}^{I}+\sum_{k=1}^{\infty} \varepsilon^{k} \overline{\mathscr{G}}_{i, \mathrm{SJ}}^{I, k}$, where the constants $C_{i}^{I}$ depend on the lower order coefficients. The $z$-independent constants $\overline{\mathscr{G}}_{i, \mathrm{SJ}}^{I, k}$ are determined from the explicit computation of the SV coefficient functions $\Delta^{I, \mathrm{SV}}\left(z, Q^{2}\right)$.

Computation of the coefficient functions $\Delta_{a}^{I}$ in perturbative QCD plays an important role in understanding the structure of hadrons. In the DIS process, the cross section factorizes into hadronic and the leptonic parts and the former can be computed by using operator product expansion in the Björken limit. Using various symmetries, the hadronic part can be expressed in terms of the structure functions $F_{1}\left(x, Q^{2}\right)$ and $F_{2}\left(x, Q^{2}\right)$. These functions factorize into calculable coefficient functions $c_{i}\left(x, Q^{2}, \mu_{F}^{2}\right), i=1,2$ and non-perturbative parton distribution functions $f_{a}\left(x, \mu_{F}^{2}\right), a=q, \bar{q}, g$. Applying the optical theorem, one relates the DIS cross section to the imaginary part of the forward scattering amplitude, where a virtual photon scatters off a nucleon. This forward scattering amplitude can be written in terms of coefficient functions $c_{i}$, where the latter can be computed by expanding in a perturbative series of the strong coupling constant. Computation of the higher-order coefficient functions [35, 36, 37, 38, 39] along with the higher order splitting functions [22, 23] and the precise measurements at DIS experiments were used to extract $F_{1}$ and $F_{2}$ accurately. Using the non-singlet part of the quark coefficient function $c_{2, q}$ computed up to three loops [14] and $c_{\phi, g}^{3}$ computed using the off-shell scalar DIS process in [15], we can extract $\Delta^{q, \mathrm{SV}}$ and $\Delta^{g, \mathrm{SV}}$, respectively, up to three-loops. Using these results and the known three-loop results for $A, B, f$ and the form factor dependent constants, we can determine $\overline{\mathscr{G}}_{i, \mathrm{SJ}}^{I, k}$ to desired accuracy in $\varepsilon$. 


\section{Results}

At the hadron colliders, the jets of quarks and gluons [40, 41, 42] capture the properties of QCD and provide insight into the IR structure of QCD processes. SCET provides a suitable platform to study the scattering or decay processes involving jet final states with small invariant masses. The corresponding observables can be factorized in terms of certain process-dependent functions such as hard functions and process-independent soft and jet functions. If we apply SCET formalism to the DIS process for the cases with $I=q, g$, we can identify the UV and IR finite parts of the form factor and SJ function with the process-dependent matching coefficient and the jet function of SCET, respectively. Note that only one massless parton initiates the hard process in each case, (for $I=q$, quark/antiquark scatters of the virtual photon in the hard process and for $I=g$, gluon scatters of off-shell scalar), there will be only one jet function in each case and hence it is straightforward to identify it with the finite part of SJ function.

The SJ function $\Phi_{\mathrm{SJ}}^{I}$ can be factorized into part containing IR poles in $\varepsilon$ and a part containing the finite terms in the limit $\varepsilon \rightarrow 0$, that is

$$
\mathscr{C} e^{2 \Phi_{\mathrm{SJ}}^{I}}=\mathscr{Z}^{I} \otimes \mathscr{C} e^{2 \Phi_{\mathrm{SJ}}^{I, \text { fin }}}
$$

where $\mathscr{Z}^{I}$ contains only IR poles in $\varepsilon$ and can be expanded as

$$
\mathscr{Z}^{I}=\delta(1-z)+\sum_{i=1}^{n} \sum_{j=1}^{2 i} a_{s}^{i} \frac{\mathscr{Z}_{i j}^{I}}{\mathcal{E}^{j}}
$$

The coefficients $\mathscr{Z}_{i j}^{I}$ expressed in terms of $A^{I}, B^{I}, f^{I}, \delta(1-z)$ and $\mathscr{D}_{i}(z)$ can be found in the original article [43]. The general expression for the jet function $\mathscr{C} e^{2 \Phi_{\mathrm{S} J}^{I, \text { fin }}}=\delta(1-z)+\left.\sum_{i=1}^{\infty} a_{s}^{i} J_{i}^{I}\right|_{k}$ up to three loops where $\left.J_{i}^{I}\right|_{k}$ represent the coefficients of $\mathscr{D}_{j}(z), \delta$ for $j \leq(2 i-1)$ can also be found in [43]. Throughout our computation, we have set $\mu_{R}^{2}=\mu_{F}^{2}=Q^{2}$. The renormalization group equation satisfied by the jet function is given by

$$
\mu_{R}^{2} \frac{d}{d \mu_{R}^{2}} J^{I}=\Gamma_{J}^{I} \otimes J^{I}
$$

where $\Gamma_{J}^{I}=\left\{B^{I}+f^{I}-A^{I} \ln \left(Q^{2} / \mu_{R}^{2}\right)\right\} \delta(1-z)-A^{I} \mathscr{D}_{0}$. We can get the logarithmic-dependent parts as well as the coefficients of $\mathscr{D}_{j}(z)$ of $J^{I}$ in terms of lower order coefficients through the above renormalization group equation in (3.3). Finally, we present the coefficients of the $\delta(1-z)$ 
parts of both quark and gluon jet functions at three-loops:

$$
\begin{aligned}
\left.J_{3}^{q}\right|_{\delta}= & C_{F}^{3}\left(274 \zeta_{3}+\frac{22}{3} \pi^{2} \zeta_{3}-\frac{400}{3} \zeta_{3}^{2}-88 \zeta_{5}+\frac{1173}{8}-\frac{3505}{72} \pi^{2}+\frac{622}{45} \pi^{4}-\frac{9871}{8505} \pi^{6}\right) \\
+ & C_{F}^{2} C_{A}\left(-\frac{28241}{27} \zeta_{3}+\frac{2200}{27} \pi^{2} \zeta_{3}+\frac{424}{3} \zeta_{3}^{2}+\frac{560}{9} \zeta_{5}+\frac{206197}{324}-\frac{17585}{72} \pi^{2}+\frac{18703}{1215} \pi^{4}\right. \\
& \left.+\frac{18703}{1215} \pi^{4}+\frac{1547}{4860} \pi^{6}\right)+C_{F}^{2} n_{f}\left(\frac{11216}{81} \zeta_{3}-\frac{136}{27} \pi^{2} \zeta_{3}+\frac{80}{3} \zeta_{5}-\frac{261587}{972}+\frac{4853}{108} \pi^{2}\right. \\
& \left.-\frac{2938}{1215} \pi^{4}\right)+C_{F} C_{A}^{2}\left(-\frac{187951}{243} \zeta_{3}+\frac{394}{9} \pi^{2} \zeta_{3}+\frac{1528}{9} \zeta_{3}^{2}-\frac{380}{9} \zeta_{5}+\frac{50602039}{52488}\right. \\
& \left.-\frac{464665}{4374} \pi^{2}+\frac{1009}{1620} \pi^{4}+\frac{221}{5103} \pi^{6}\right)+C_{F} C_{A} n_{f}\left(\frac{7414}{81} \zeta_{3}-\frac{32}{9} \pi^{2} \zeta_{3}+\frac{16}{3} \zeta_{5}-\frac{2942843}{13122}\right. \\
+ & \left.\frac{68324}{2187} \pi^{2}-\frac{209}{405} \pi^{4}\right)+C_{F} n_{f}^{2}\left(\frac{376}{243} \zeta_{3}+\frac{124903}{13122}-\frac{466}{243} \pi^{2}+\frac{2}{45} \pi^{4}\right) \\
\left.J_{3}^{g}\right|_{\delta} & =C_{A}^{3}\left(-\frac{452770}{243} \zeta_{3}+\frac{1364}{9} \pi^{2} \zeta_{3}+\frac{1600}{9} \zeta_{3}^{2}-44 \zeta_{5}+\frac{55853711}{26244}-\frac{2055109}{4374} \pi^{2}\right. \\
& \left.+\frac{53633}{1620} \pi^{4}-\frac{16309}{20412} \pi^{6}\right)+C_{A}^{2} n_{f}\left(\frac{2734}{9} \zeta_{3}-\frac{88}{9} \pi^{2} \zeta_{3}+\frac{208}{9} \zeta_{5}-\frac{17323633}{26244}\right. \\
& \left.+\frac{330062}{2187} \pi^{2}-\frac{18727}{2430} \pi^{4}\right)+C_{A} n_{f}^{2}\left(-\frac{1004}{243} \zeta_{3}+\frac{1613639}{26244}-\frac{3656}{243} \pi^{2}+\frac{506}{1215} \pi^{4}\right) \\
& +C_{A} C_{F} n_{f}\left(\frac{21200}{81} \zeta_{3}-\frac{160}{9} \pi^{2} \zeta_{3}+\frac{584}{9} \zeta_{5}-\frac{389369}{972}+\frac{712}{27} \pi^{2}+\frac{76}{405} \pi^{4}\right) \\
& +C_{F}^{2} n_{f}\left(\frac{148}{3} \zeta_{3}-80 \zeta_{5}+\frac{143}{9}\right)+C_{F} n_{f}^{2}\left(-\frac{104}{3} \zeta_{3}+\frac{7001}{162}-\frac{10}{9} \pi^{2}\right) \\
& +n_{f}^{3}\left(-\frac{1000}{729}+\frac{40}{81} \pi^{2}\right) \cdot
\end{aligned}
$$

Our result for the quark jet function (3.4) provides an independent check on the more recent calculation completed using SCET framework [11] and the result for the gluon jet function (3.5) is a new result originating from our work.

\section{Conclusion}

In this article, we have shown how one of the building blocks of SCET, namely, the jet function can be related to the well-known coefficient function of the DIS cross section. This novel connection provides an alternate and elegant way to obtain both quark and gluon jet functions order by order in pQCD from the known coefficient functions. While we confirm the three-loop quark jet function reported recently in [11], the three-loop gluon jet function presented in the article is a new result. The important ingredient to obtain these results is the parton coefficient functions [14, 15] of DIS process up to the three-loop level in QCD. We have used the factorization properties of scattering cross section and exploited universal structure of soft and collinear dynamics to relate soft plus jet function of DIS against the jet function in SCET. The three-loop quark and gluon jet functions are the important ingredients to the $\mathrm{N}$-jettiness IR subtraction method [44, 45] at $\mathrm{N}^{3} \mathrm{LO}$ 
and to threshold resummation up to $\mathrm{N}^{3} \mathrm{LL}^{\prime}$ in the SCET framework to study processes involving final state jets. Thanks to the wealth of precise predictions in perturbative QCD for various important observables, one can unfold the underlying universal infrared structure QCD amplitudes and determine process-independent building blocks that capture infrared dynamics of high-energy scattering processes.

\section{References}

[1] C. W. Bauer, S. Fleming and M. E. Luke, Summing Sudakov logarithms in $B \longrightarrow X($ s gamma $)$ in effective field theory, Phys. Rev. D63 (2000) 014006, [hep-ph/ 0005275 ].

[2] C. W. Bauer, S. Fleming, D. Pirjol and I. W. Stewart, An Effective field theory for collinear and soft gluons: Heavy to light decays, Phys. Rev. D63 (2001) 114020, [hep-ph/ 0011336 ].

[3] C. W. Bauer and I. W. Stewart, Invariant operators in collinear effective theory, Phys. Lett. B516 (2001) 134-142, [hep-ph/0107001].

[4] C. W. Bauer, D. Pirjol and I. W. Stewart, Soft collinear factorization in effective field theory, Phys. Rev. D65 (2002) 054022, [hep-ph/ 010904 5].

[5] C. W. Bauer, S. Fleming, D. Pirjol, I. Z. Rothstein and I. W. Stewart, Hard scattering factorization from effective field theory, Phys. Rev. D66 (2002) 014017, [hep-ph/ 0202088 ].

[6] M. Beneke, A. P. Chapovsky, M. Diehl and T. Feldmann, Soft collinear effective theory and heavy to light currents beyond leading power, Nucl. Phys. B643 (2002) 431-476, [hep-ph/ 0206152 ].

[7] Y. Li, A. von Manteuffel, R. M. Schabinger and H. X. Zhu, $N^{3}$ LO Higgs boson and Drell-Yan production at threshold: The one-loop two-emission contribution, Phys. Rev. D90 (2014) 053006, [1404.5839].

[8] C. W. Bauer and A. V. Manohar, Shape function effects in $B \rightarrow X(s)$ gamma and $B \rightarrow X(u) l$ anti-nu decays, Phys. Rev. D70 (2004) 034024, [hep-ph/ 0312109 ].

[9] S. W. Bosch, B. O. Lange, M. Neubert and G. Paz, Factorization and shape function effects in inclusive B meson decays, Nucl. Phys. B699 (2004) 335-386, [hep-ph/ 0402094 ].

[10] T. Becher and M. Neubert, Toward a NNLO calculation of the anti-B $\rightarrow X(s)$ gamma decay rate with a cut on photon energy. II. Two-loop result for the jet function, Phys. Lett. B637 (2006) 251-259, [hep-ph/0603140].

[11] R. Brüser, Z. L. Liu and M. Stahlhofen, Three-Loop Quark Jet Function, Phys. Rev. Lett. 121 (2018) 072003, [1804.09722].

[12] T. Becher and M. D. Schwartz, Direct photon production with effective field theory, JHEP 02 (2010) 040, [0911.0681].

[13] T. Becher and G. Bell, The gluon jet function at two-loop order, Phys. Lett. B695 (2011) 252-258, [1008.1936].

[14] J. A. M. Vermaseren, A. Vogt and S. Moch, The Third-order QCD corrections to deep-inelastic scattering by photon exchange, Nucl. Phys. B724 (2005) 3-182, [hep-ph/ 0504242 ].

[15] G. Soar, S. Moch, J. A. M. Vermaseren and A. Vogt, On Higgs-exchange DIS, physical evolution kernels and fourth-order splitting functions at large $x$, Nucl. Phys. B832 (2010) 152-227, [0912.0369]. 
[16] V. Ravindran, On Sudakov and soft resummations in QCD, Nucl. Phys. B746 (2006) 58-76, [hep-ph/0512249].

[17] V. Ravindran, Higher-order threshold effects to inclusive processes in QCD, Nucl. Phys. B752 (2006) 173-196, [hep-ph/0603041].

[18] V. Ravindran, J. Smith and W. L. van Neerven, QCD threshold corrections to di-lepton and Higgs rapidity distributions beyond $N^{2}$ LO, Nucl. Phys. B767 (2007) 100-129, [hep-ph/ 0608308 ].

[19] P. Banerjee, G. Das, P. K. Dhani and V. Ravindran, Threshold resummation of the rapidity distribution for Higgs production at NNLO+NNLL, Phys. Rev. D97 (2018) 054024, [1708 . 05706].

[20] P. Banerjee, G. Das, P. K. Dhani and V. Ravindran, Threshold resummation of the rapidity distribution for Drell-Yan production at NNLO+NNLL, Phys. Rev. D98 (2018) 054018, [1805.01186].

[21] K. G. Chetyrkin, B. A. Kniehl and M. Steinhauser, Decoupling relations to O (alpha-s**3) and their connection to low-energy theorems, Nucl. Phys. B510 (1998) 61-87, [hep-ph/9708255].

[22] S. Moch, J. A. M. Vermaseren and A. Vogt, The Three loop splitting functions in QCD: The Nonsinglet case, Nucl. Phys. B688 (2004) 101-134, [hep-ph/ 0403192 ].

[23] A. Vogt, S. Moch and J. A. M. Vermaseren, The Three-loop splitting functions in QCD: The Singlet case, Nucl. Phys. B691 (2004) 129-181, [hep-ph/0 404111$].$

[24] J. Davies, A. Vogt, B. Ruijl, T. Ueda and J. A. M. Vermaseren, Large- $n_{f}$ contributions to the four-loop splitting functions in QCD, Nucl. Phys. B915 (2017) 335-362, [1610 . 07477].

[25] V. V. Sudakov, Vertex parts at very high-energies in quantum electrodynamics, Sov. Phys. JETP 3 (1956) 65-71.

[26] A. H. Mueller, On the Asymptotic Behavior of the Sudakov Form-factor, Phys. Rev. D20 (1979) 2037.

[27] J. C. Collins, Algorithm to Compute Corrections to the Sudakov Form-factor, Phys. Rev. D22 (1980) 1478.

[28] A. Sen, Asymptotic Behavior of the Sudakov Form-Factor in QCD, Phys. Rev. D24 (1981) 3281.

[29] S. Moch, J. A. M. Vermaseren and A. Vogt, The Quark form-factor at higher orders, JHEP 08 (2005) 049, [hep-ph/0507039].

[30] S. Catani and L. Trentadue, Resummation of the QCD Perturbative Series for Hard Processes, Nucl. Phys. B327 (1989) 323-352.

[31] S. Catani and L. Trentadue, Comment on QCD exponentiation at large x, Nucl. Phys. B353 (1991) 183-186.

[32] A. Vogt, Next-to-next-to-leading logarithmic threshold resummation for deep inelastic scattering and the Drell-Yan process, Phys. Lett. B497 (2001) 228-234, [hep-ph/ 0010146$].$

[33] V. Ravindran, J. Smith and W. L. van Neerven, Two-loop corrections to Higgs boson production, Nucl. Phys. B704 (2005) 332-348, [hep-ph/ 0408315$].$

[34] O. V. Tarasov, A. A. Vladimirov and A. Yu. Zharkov, The Gell-Mann-Low Function of QCD in the Three Loop Approximation, Phys. Lett. B93 (1980) 429-432.

[35] W. L. van Neerven and E. B. Zijlstra, Order alpha-s**2 contributions to the deep inelastic Wilson coefficient, Phys. Lett. B272 (1991) 127-133.

[36] E. B. Zijlstra and W. L. van Neerven, Order alpha-s**2 correction to the structure function F3 ( $x$, $Q^{* * 2}$ ) in deep inelastic neutrino - hadron scattering, Phys. Lett. B297 (1992) 377-384. 
[37] E. B. Zijlstra and W. L. van Neerven, Contribution of the second order gluonic Wilson coefficient to the deep inelastic structure function, Phys. Lett. B273 (1991) 476-482.

[38] E. B. Zijlstra and W. L. van Neerven, Order alpha-s**2 QCD corrections to the deep inelastic proton structure functions F2 and F(L), Nucl. Phys. B383 (1992) 525-574.

[39] R. Hamberg, W. L. van Neerven and T. Matsuura, A complete calculation of the order $\alpha-s^{2}$ correction to the Drell-Yan K factor, Nucl. Phys. B359 (1991) 343-405.

[40] G. F. Sterman and S. Weinberg, Jets from Quantum Chromodynamics, Phys. Rev. Lett. 39 (1977) 1436.

[41] G. P. Salam and G. Soyez, A Practical Seedless Infrared-Safe Cone jet algorithm, JHEP 05 (2007) 086, [0704.0292].

[42] M. Cacciari, G. P. Salam and G. Soyez, The Anti-k(t) jet clustering algorithm, JHEP 04 (2008) 063, [0802.1189].

[43] P. Banerjee, P. K. Dhani and V. Ravindran, Gluon jet function at three loops in QCD, Phys. Rev. D98 (2018) 094016, [1805.02637].

[44] J. Gaunt, M. Stahlhofen, F. J. Tackmann and J. R. Walsh, N-jettiness Subtractions for NNLO QCD Calculations, JHEP 09 (2015) 058, [1505.04794].

[45] R. Boughezal, C. Focke, X. Liu and F. Petriello, W-boson production in association with a jet at next-to-next-to-leading order in perturbative QCD, Phys. Rev. Lett. 115 (2015) 062002, [1504.02131]. 